\title{
Elevated expression of Derlin-1 associates with unfavorable survival time of squamous cell carcinoma of the head and neck and promotes its malignance
}

\author{
Leiming Pi ${ }^{1}$, Gangcai Zhu ${ }^{2}$, Li She ${ }^{1}$, Ming Wei ${ }^{1}$, Guancheng Liu ${ }^{1}$, Changhan Chen ${ }^{1}$, Di Hu${ }^{1}$, Fusen Peng ${ }^{3}$,

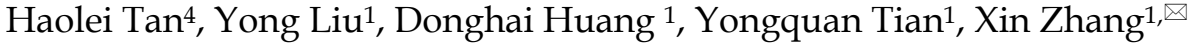 \\ 1. Department of Otolaryngology Head and Neck Surgery, Xiangya Hospital, Central South University, 87 Xiangya Road, Changsha 410008, Hunan, People's \\ Republic of China \\ 2. Department of Otolaryngology Head and Neck Surgery, The Second Xiangya Hospital, Central South University, 139 Renmin Road, Changsha 410010, \\ Hunan, People's Republic of China \\ 3. Department of Otolaryngology Head and Neck Surgery, Loudi Central Hospital, Loudi, Hunan, People's Republic of China \\ 4. Department of Head and Neck Surgery, Hunan Cancer Hospital, The Affiliated Tumor Hospital of Xiangya Medical School, Central South University, 283 \\ Tongzipo Road, Changsha, Hunan 410013, People's Republic of China \\ $\square$ Corresponding author: Xin Zhang, E-mail: xinzhang@csu.edu.cn \\ (c) Ivyspring International Publisher. This is an open access article distributed under the terms of the Creative Commons Attribution (CC BY-NC) license \\ (https:// creativecommons.org/licenses/by-nc/4.0/). See http://ivyspring.com/terms for full terms and conditions.
}

Received: 2017.01.30; Accepted: 2017.05.03; Published: 2017.07.21

\begin{abstract}
Derlin-1 is over-expressed to function as an oncoprotein in breast, lung and colon cancers. However, the implications of Derlin-1 involved in squamous cell carcinoma of the head and neck $(\mathrm{SCCHN})$ remain unknown. This study aims to investigate the effects of Derlin-1 expression on SCCHN tissues and cells. The potential mechanism of Derlin-1 regulating SCCHN cell proliferation, apoptosis and metastasis was also indicated in this work. Western blot and immunohistochemistry $(\mathrm{IHC})$ assays showed that Derlin-1 was over-expressed in $114 \mathrm{SCCHN}$ samples and five SCCHN cell lines. We found that the expression of Derlin-1 was positively associated with lymph node metastasis, clinical stage and recurrence in our SCCHN patients' samples. Survival analysis indicated that high expression of Derlin-1 was significantly associated with shorter overall survival (OS) and disease-free survival (DFS). Knock down expression of Derlin-1 in SCCHN cell lines was found to inhibit cell proliferation, metastasis and promote cell apoptosis. Further experiments showed that signals of PI3K/Akt, p53 and Smad2/3 may involve in these processes. In all, Derlin-1 might be a novel prognostic marker of SCCHN patients and plays an oncogenic role in $\mathrm{SCCHN}$ cell progression.
\end{abstract}

Key words: SCCHN, Derlin-1, Survival, Apoptosis, Metastasis, EMT

\section{Introduction}

Squamous cell carcinoma of the head and neck (SCCHN) is the sixth most prevalent malignancy worldwide involving in the nasal cavities, paranasal sinuses, oral cavity, nasopharynx, oropharynx, hypopharynx, and larynx ${ }^{1}$. Despite the multimodal therapies including surgery, radiotherapy, and chemotherapy have been applied in clinic for decades, the quality of life and survival rate in SCCHN patients lag behind people's expectation ${ }^{2}$. Patients may get absolutely different outcome even them belong to the same TNM classification. There is a large limitation for physician to predict patient outcome and make therapeutic decisions based only on TNM classification. Molecular tumor biomarkers facilitate the diagnosis and prognosis for SCCHN patients ${ }^{3}$. Therefore, patient may be benefited from some novel biomarkers involved in metastasis and pro-apoptosis.

Derl-like-protein-1 (Derlin-1) is a human homologue of yeast Der1p containing four transmembrane regions within the lipid bilayer of endoplasmic reticulum membrane ${ }^{4}$. It was initially reported as a partner of the p97 ATPase complex and regulator of 
misfolded protein degradation from the endoplasmic reticulum (ER) to cytosolic ${ }^{5}$. The ER is a specialized organelle where cells correct protein folding and modify secretory and membrane proteins ${ }^{6}$. Cancer cellular adaptation to ER stress is mediated by the unfolded protein response (UPR), when cancer cell under intrinsic and external adverse conditions such as hypoxia or chemo-radiotherapy ${ }^{6,7}$. There were evidences showed that the Derlin-1 played crucial roles in cystic fibrosis and cancers such as breast ${ }^{8}$, colon $^{9}$, bladder ${ }^{10}$ and lung ${ }^{11}$ cancers. However, there is nearly no study about Derlin-1 in SCCHN. Here, we found that Derlin-1 was over-expressed in SCCHN samples comparing to adjacent normal tissues. The expression of Derlin-1 was associated with T-stage, lymph node metastasis and clinical stage. And high expression of Derlin-1 independently predicted a shorter survival time of SCCHN patients. Knock down Derlin-1 expression regulated cell proliferation, apoptosis and migration in two SCCHN cell lines. Furthermore, the classical signal proteins, like p53, Smad2/3, PI3K/Akt, may involve in the regulation of SCCHN by Derlin-1. In general, this study may identify a promising biomarker for survival prediction and provide some novel insights in the mechanism of SCCHN malignance.

\section{Materials and Methods}

\section{Tissues and follow-up}

All sections of formalin-fixed paraffin-embedded SCCHN samples were from 114 patients who were treated at Xiangya Hospital from January 2000 to October 2005. In addition, 18 grossly dissected pieces of tumor and adjacent normal tissues were acquired from September 2012 to February 2013 and stored at $-80^{\circ} \mathrm{C}$ until Western blot analysis. Consent for use of these samples was obtained from the Ethics Committee of the Xiangya Hospital of Central South University. All selected patients did not have a history of previous malignancies, radiotherapy or chemotherapy. The pathological characteristics of all the patients including age, differentiation, TNM information, smoking and alcohol history are summarized in Table 1.

Follow-up materials were obtained from 108 patients after surgery (follow-up rate was $94.7 \%$ ). Six patients lost connection because of telephone number or residence changes. Recurrence was checked by clinical examination, imaging evaluation and pathological studies. Overall survival (OS) and disease-free (DFS) survival were calculated from the date of surgery to the date of death or tumour relapse respectively. Deaths from other causes were treated as censored cases. The follow-up time ranged from two to sixty months.
Table 1: Correlations between Derlin-1 expression and clinicopathological variables in patients with SCCHN

\begin{tabular}{|c|c|c|c|c|c|c|}
\hline \multirow[t]{2}{*}{ Variables } & & \multirow{2}{*}{$\begin{array}{l}\text { Number } \\
\text { (percentage) }\end{array}$} & \multicolumn{2}{|c|}{ Derlin-1 expression } & \multirow[t]{2}{*}{$\mathrm{X}^{2}$ Value } & \multirow[t]{2}{*}{ P Value } \\
\hline & & & high & low & & \\
\hline \multirow[t]{2}{*}{ age } & $\leq 57$ & $52(45.61 \%)$ & 22 & 30 & 0.018 & 1 \\
\hline & $>57$ & $62(54.39 \%)$ & 27 & 35 & & \\
\hline \multirow[t]{2}{*}{ Gender } & Male & $109(95.61 \%)$ & 47 & 62 & 0.019 & 0.890 \\
\hline & Female & $5(4.39 \%)$ & 2 & 3 & & \\
\hline \multirow[t]{2}{*}{ Alcohol intake } & No & $47(41.23 \%)$ & 22 & 25 & 0.478 & 0.566 \\
\hline & Yes & $67(58.77 \%)$ & 27 & 40 & & \\
\hline \multirow[t]{2}{*}{ Tobacco use } & No & $30(26.32 \%)$ & 11 & 19 & 0.663 & 0.520 \\
\hline & Yes & $84(73.68 \%)$ & 38 & 46 & & \\
\hline \multirow[t]{2}{*}{ Grade } & $\mathrm{G} 2+\mathrm{G} 3$ & $39(34.21 \%)$ & 18 & 21 & 0.243 & 0.692 \\
\hline & G1 & $75(65.79 \%)$ & 31 & 44 & & \\
\hline \multirow[t]{2}{*}{ Tumor position } & Glottic & $81(71.05 \%)$ & 32 & 49 & 1.38 & 0.298 \\
\hline & Others & $33(28.95 \%)$ & 17 & 16 & & \\
\hline \multirow[t]{2}{*}{ T-stage } & $\mathrm{T} 1+\mathrm{T} 2$ & $63(55.26 \%)$ & 25 & 38 & 0.626 & 0.452 \\
\hline & $\mathrm{T} 3+\mathrm{T} 4$ & $51(44.74 \%)$ & 24 & 27 & & \\
\hline \multirow[t]{2}{*}{ Clinical stage } & I-II & $66(57.89 \%)$ & 19 & 47 & 12.887 & 0.001 \\
\hline & III-IV & $48(42.11 \%)$ & 30 & 18 & & \\
\hline \multirow{2}{*}{$\begin{array}{l}\text { Lymph node } \\
\text { status }\end{array}$} & N- & $84(73.68 \%)$ & 29 & 55 & 9.319 & 0.003 \\
\hline & $\mathrm{N}+$ & $30(26.32 \%)$ & 20 & 10 & & \\
\hline \multirow[t]{2}{*}{ Recurrence $\$$} & No & $56(51.85 \%)$ & 9 & 47 & 31.349 & $<0.001$ \\
\hline & Yes & $52(48.15 \%)$ & 36 & 16 & & \\
\hline
\end{tabular}

Note: $\mathrm{p}$ value $<0.05$ was considered to be statistically significant and labeled in bold font

$\S$ : Six patients lost to follow-up because of telephone number changes or home moving.

\section{Immunohistochemistry and evaluation of Derlin-1}

Immunohistochemistry (IHC) was performed as we previously reported ${ }^{12}$. Briefly, sections were deparaffinized, subjected to antigen retrieval. All slides were then soaked for a minimum of 5 minutes in phosphate-buffered saline (PBS), followed by incubation with normal immunoglobulin $G(\operatorname{IgG})$ for 15 minutes and anti-Derlin-1 antibody (Sigma, USA $1: 1000)$ at $4^{\circ} \mathrm{C}$ overnight. The slides were then rinsed with PBS three times and incubated with HRPlabeled goat anti-rabbit IgG. After being washed in PBS three times, the slides were stained with diaminobenzidine chromagen, and counterstained with hematoxylin.

Slides were visualized with light microscopy and qualitatively scored by two pathologists who were blinded to clinicalpathological variables. An immunohistochemical intensity score (a) for Derlin-1 expression was empirically determined ranging from none (0), weak (1), moderate (2) to strong (3). In addition, the percentage of cell labeling was scored (b) as less than $25 \%$ (1), $25-50 \%$ (2), $50-75 \%$ (3) or greater than $75 \%$ (4). The total score of each slide was calculated by a multiplied by $b$. The determination of Derlin-1 expression into high or low is based on a 
cut-off that is best for predicting overall survival status of SCCHN patients (the detailed analysis was showed in statistic section).

\section{Cell lines and Transfection}

DOK is immortalized non-malignant cell line derived from human oral mucosa. 5-8F and 6-10B derived from human nasopharyngeal carcinoma. $\mathrm{FaDu}$ and SCC-10A derived from human hypopharynx and larynx respectively. Tu686 derived from human oropharynx. Tu686, FaDu and SCC-10A were grown in Dulbecco's minimal essential medium containing $10 \%$ fetal bovine serum and 50 units $/ \mathrm{mL}$ penicillin and $50 \mu \mathrm{g} / \mathrm{mL}$ streptomycin sulfate. DOK, $5-8 \mathrm{~F}$ and 6-10B were grown in RPMI Medium 1640 containing $10 \%$ fetal bovine serum and 50 units $/ \mathrm{mL}$ penicillin and $50 \mu \mathrm{g} / \mathrm{mL}$ streptomycin sulfate. Tu686 cell line was a gift kindly from Georgia Chen (Emory University). 5-8F, 6-10B, FaDu and SCC-10A cell lines were purchased from Cell Biology Research Laboratory of Central South University. Cells were incubated at $37^{\circ} \mathrm{C}$ in a humidified atmosphere of $5 \%$ $\mathrm{CO}_{2}$. Cells were used in experiments when they were in logarithmic phase.

Derlin-1 shRNA was purchased from GeneCopoeis, Inc. The effectiveness of Derlin-1 shRNA had been confirmed by western blot. Lipofectamine 2000 (Invitrogen Corporation) was used in transient transfection with Derlin-1 shRNA. Following transfection, cells were incubated for 48 hours before being harvested for assays.

\section{Western blot analysis}

Thirty micrograms of total protein were separated by $10 \%$ SDS-PAGE as previously described $^{13}$ and then transferred onto polyvinylidene difluoride membranes (Millipore, Bedford, MA). The blotted membranes were incubated with rabbit antibody against Derlin-1 (A81438, dilution 1:1000) (Sigma, USA), p53 (ab131442, Abcam), PI3K (3011S, Cell Signaling Technology), p-PI3K (Tyr458) (Tyr199) (4228S, Cell Signaling Technology), p-AKT (Ser 473) (4060P, Cell Signaling Technology), AKT (4685S, Cell Signaling Technology), Vimentin (10366-1-AP, Proteintech), E-cadherin, (20874-1-AP, Proteintech), Smad2/3 Antibody Sampler Kit (12747, Cell Signaling Technology), at $4^{\circ} \mathrm{C}$ overnight. After washing with PBS, they were incubated with HRP-labelled Goat Anti-rabbit IgG for $1 \mathrm{~h}$ at room temperature. $\beta$-actin (Beyotime, China, dilution 1:1000) was incubated as internal control. The experiment was repeated in triplicates.

\section{Cell viability and flows cytometry}

Following transient transfection for $48 \mathrm{~h}$, the shRNA control or Derlin-1 shRNA transfected cells were cultured in 96-well plates for 96h. At each time, cells were stained with Cell Counting Kit-8 (Beyotime, China) for investigating the cell numbers according to the manufacturer's instructions. The absorbance (490nm) was measured by a microplate reader. The growth curves for cell line with shRNA control and Derlin-1 shRNA was illustrated by GraphPad Prism software.

The shRNA control or Derlin-1 shRNA transfected cells were cultured in 6-well plates for $48 \mathrm{~h}$. Apoptotic cells were determined by phycoerythrin (PE) and 7-AAD staining in flow cytometry. Briefly, replicate cultures of $1 \times 10^{6}$ cells were plated in cell culture wells. The cells were transfected with control shRNA or Derlin-1shRNA. The PE and 7-AAD staining fluorescence of individual nuclei was measured in the red fluorescence using a flow cytometer (Beckman Coulter Elite; Beckman Coulter, Fullerton, CA, USA), and the data were registered in a logarithmic scale.

\section{Cell wound and Transwell migration assays}

Transfected cells were cultured for $48 \mathrm{~h}$, and seeded in 6-well plates were permitted to proliferate to near $100 \%$ saturation adherent density and then were wounded by removing a line of cells with a disinfected Eppendorf Tip. 48 hours later, the wounded areas were photographed under a microscope.

Following transient transfection for $48 \mathrm{~h}$, all kinds of transfected cells were plated in Transwell cell culture inserts (Corning Costar) at a concentration of $2.5 \times 10^{4}$ cells/well. Then, the cells were maintained and allowed to migrate for 48 hours. The cells that had not invaded were removed from the upper surface using a cotton swab. The cells that had invaded to the lower surface were stained with crystal violet solution and photographed under the microscope.

\section{Statistical analysis}

All statistical analyses were performed using IBM SPSS statistical software, version 21.0 (SPSS Inc., Chicago, IL. USA). Continuous variables were expressed as mean $\pm \mathrm{SD}$. The chi-square test was used to analyze the correlation between Derlin-1 expression and clinicopathological features. The Kaplan-Meier method and log-rank test were applied for survival. Identification of relevant prognostic factors was performed by univariate and multivariate Cox regression analysis. The best cut-off for Derlin-1 high expression determination was based on its total staining score for predicting our $50 \%$ overall survival status by receiver operating characteristic (ROC) curve. Differences were considered to be significant when $\mathrm{P}<0.05$. All tests were two-sided. 


\section{Results}

\section{The expression of Derlin-1 was elevated in SCCHN cancers tissues and cell lines}

In order to investigate the expression profile of Derlin-1 in SCCHN, 18 pairs of fresh cancer tissue and adjacent mucosa were used in the western blot experiment. The expression of Derlin-1 was much higher in cancer than adjacent mucosa in 18 pairs of patient samples (Figure 1a). Derlin-1 expression was also measured in 5 kinds of SCCHN cancer cell lines

a

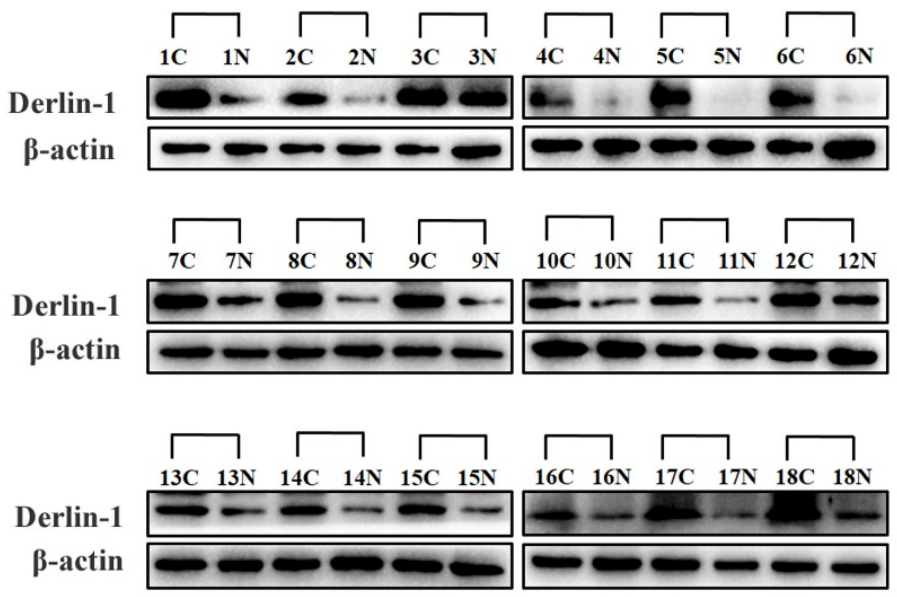

b

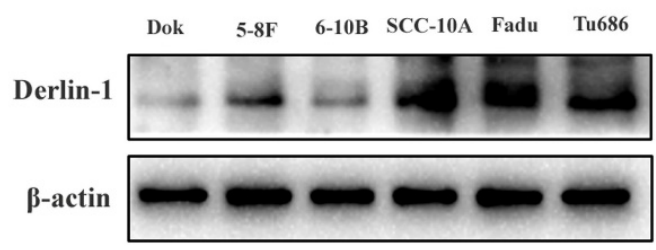

c

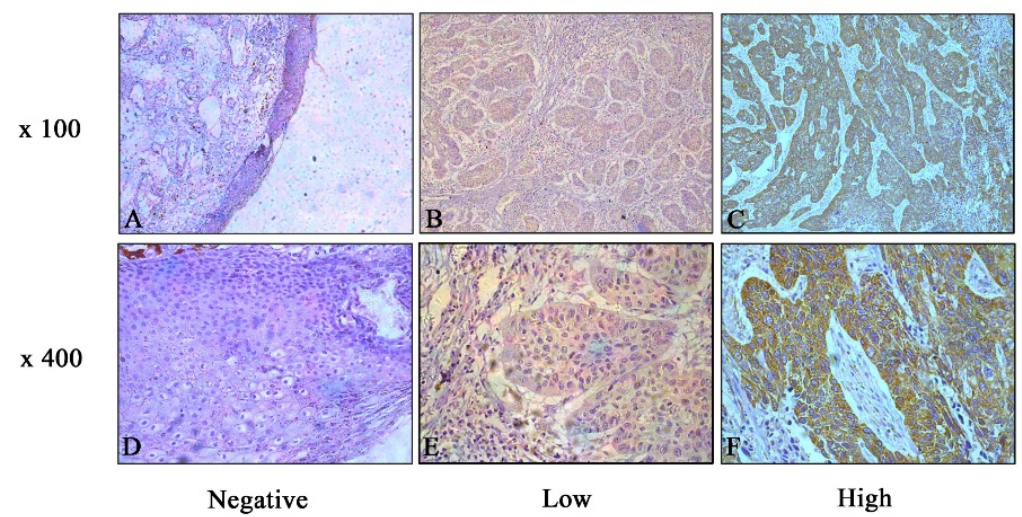

Figure 1: The expression of Derlin-1 in SCCHN cell lines and tissues. (a). Western blotting of Derlin-1 protein expression in the 18 pairs of primary human SCCHN cancer tissues (C) with adjacent normal mucosa (N). (b). The Derlin-1 expression in 5 SCCHN cell lines and 1 (5-8F, 6-10B, SCC-10A, $\mathrm{FaDu}, \mathrm{Tu686}$ ) and an immortalized non-malignant cell line derived from oral mucosa(DOK). (c). Representative immunohistochemical staining for Derlin-1 in primary human SCCHN tissues. Negative stain was detected in adjacent normal mucosa (Left panel); Low expression of Derlin-1 protein in primary SCCHN specimens (middle panel); High expression of Derlin-1 protein in primary SCCHN specimens (right panel); original magnification: Top row, $\times 100$; bottom row, $\times 400$.
(5-8F, 6-10B, SCC-10A, FaDu, Tu686) and an immortalized non-malignant cell line derived from oral mucosa (DOK). As showed in Figure 1b, Derlin-1 expressed much more in SCCHN cell lines than non-malignant cell line. Among the SCCHN cell lines, the Delrin-1 expression in 5-8F and 6-10B was a bit lower than SCC-10A, Tu686 and FaDu.

In addition, IHC analysis was also performed to check the relative expression of Derlin-1 protein in 114 paraffin-embedded SCCHN slides and 6 adjacent normal mucosae. There was no stain of Delrin-1 in normal mucosa tissues (Figure 1c, left panel), but positively stained in 94.7\% (108/114) of cancer slides. Among the patients with positive stain of Derlin-1, $45.4 \%(49 / 108)$ of patients were detected with high expression of Derlin-1 (Figure 1c, middle and right panel).

\section{The Derlin-1 expression correlated with clinicopathological parameters in SCCHN patients}

Given that Derlin-1 expression was elevated in SCCHN cancer samples and cell lines based on above results, the association of Derlin-1 expression with clinicopathological parameter was analyzed in 114 SCCHN patients. As summarized in Table 1, Derlin-1 expression was significantly associated with neck lymph node metastasis $(p=0.003)$, recurrence $(p$ $<0.001)$ and clinical stage $(\mathrm{p}=0.001)$ and respectively. However, no significant relationship was observed between Derlin-1 protein level and other factors such as age $(\mathrm{p}=1.000)$ and primary tumor site $(\mathrm{p}=$ $0.298)$.

\section{The expression of Derlin-1 is an independent prognostic factor of disease-free survival time in SCCHN patients}

Among the 114 SCCHN patients, 108 were followed up. As showed in Figure 2, Kaplan-Meier survival analysis implied that patients with high expression of Derlin-1 seemed to suffer lower possibility of five-year overall survival (OS: $24.09 \%$ versus $84.13 \%$ ) and disease-free survival (DFS: $18.07 \%$ versus $74.07 \%$ ). A log-rank test further confirmed that a significant difference between five-year DSF and OS rate $(\mathrm{p}<0.01)$. 

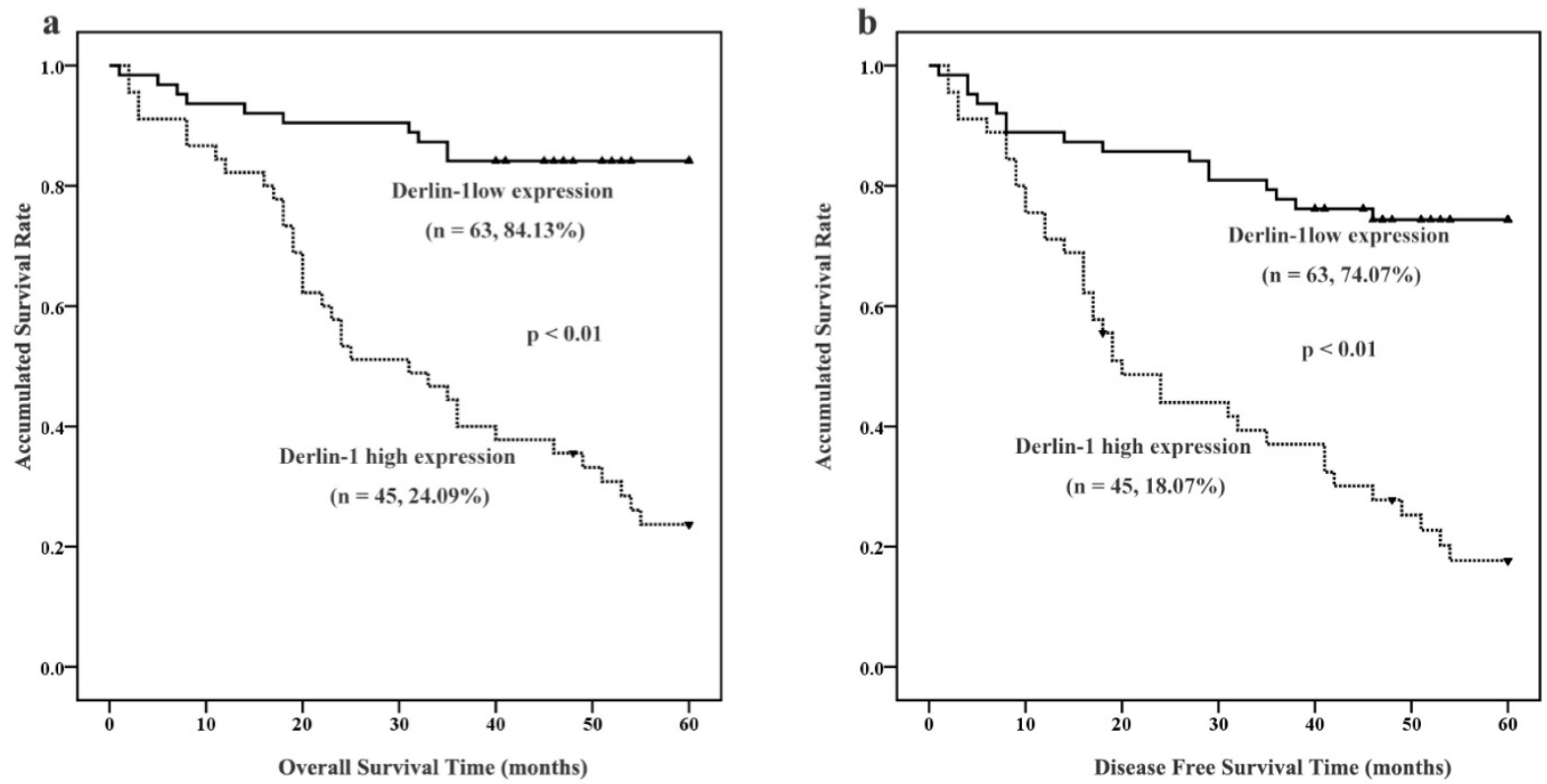

Figure 2: The expression of Derlin-1 associated with prognosis of SCCHN patients. (a, b). Kaplan-Meier survival analysis of overall survival and disease-free survival in all patients with SCCHN was generated according to Derlin-1 protein expression. The log-rank test was applied to calculate $p$ value. Number of patients and five-year survival rate was indicated in brace. solid triangle represented censored data.

Univariate and multivariate Cox regression analyses were employed to clarify the independent factors affecting OS and DFS in SCCHN patients. Firstly, tobacco smoking, clinical stage, tumor classification, tumor position, lymph node metastasis and Derlin-1 protein expression were significantly associated with both OS and DFS after screening all the parameters in Table 2 by univariate analysis. Therefore, tobacco smoking, clinical stage, tumor classification, tumor position, lymph node metastasis and Derlin-1 protein expression were put into multivariate Cox analyses to see which one is independent factor impacting patient outcome. Tobacco smoking and Derlin-1 protein expression were finally determined to be independent prognostic factors for both OS and DFS in SCCHN patients by the multivariate Cox analyses with forward stepwise selection (Table 2).

\section{Decreased expression of Derlin-1 reduced proliferation of SCCHN cell lines}

These above results indicated high expression of Derlin-1 correlated with poor prognosis and advanced tumor classification. To understand the possible effect of Derlin-1 on SCCHN cells, shRNA of Derlin-1 was transferred into Tu686 and FaDu cell line. Western blot result revealed that Derlin-1 protein was successfully reduced (Figure 3a). CCK8 assay showed that there was significant decrease in cell viability of Tu686 and FaDu when Derlin-1 was knock down (Figure 3b-c). Meanwhile, the phosphorylated PI3K and Akt were decreased in the Derlin-1 knock group (Figure 3d-g).
Table 2: Univariate and multivariate COX analysis in SCCHN patients

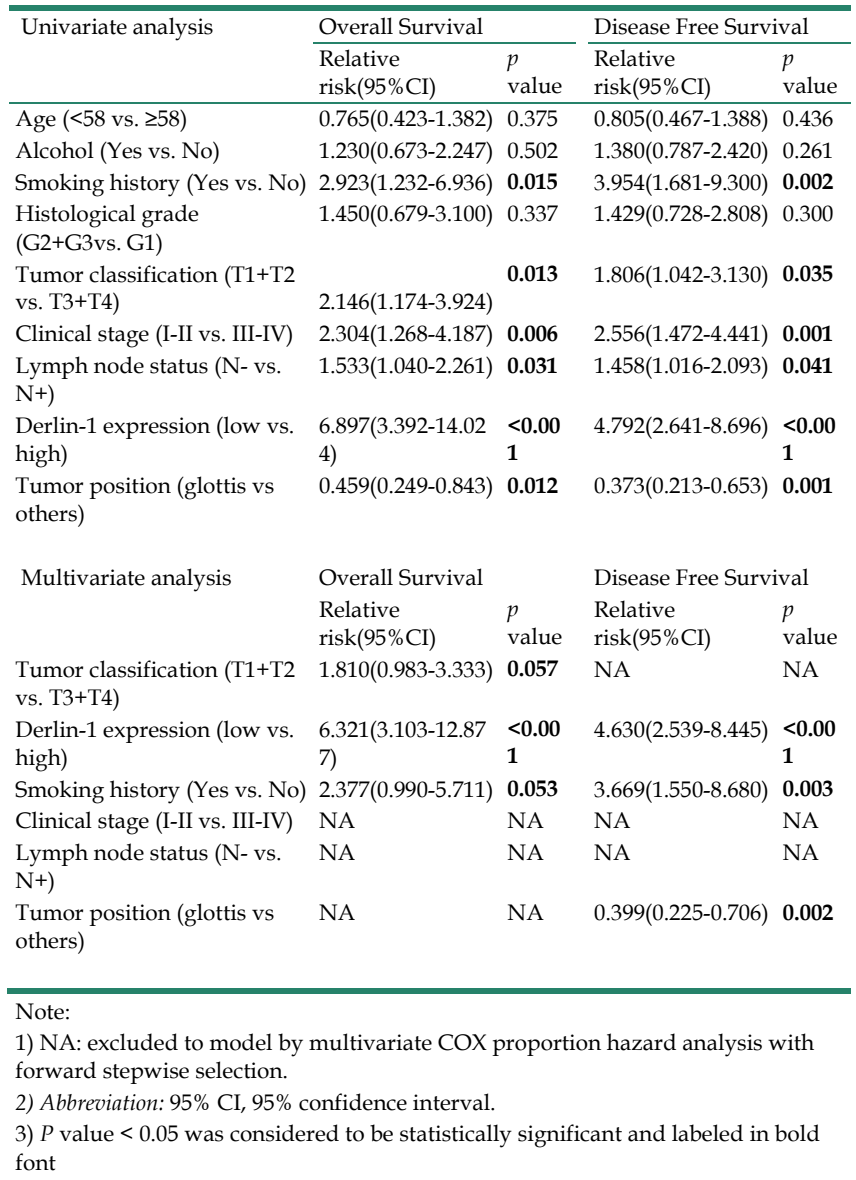


a

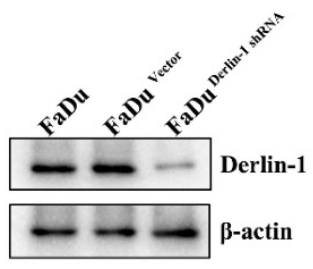

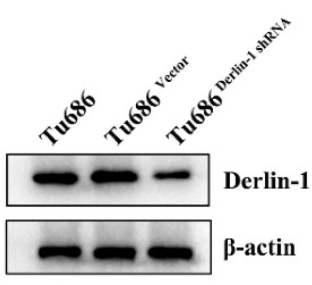

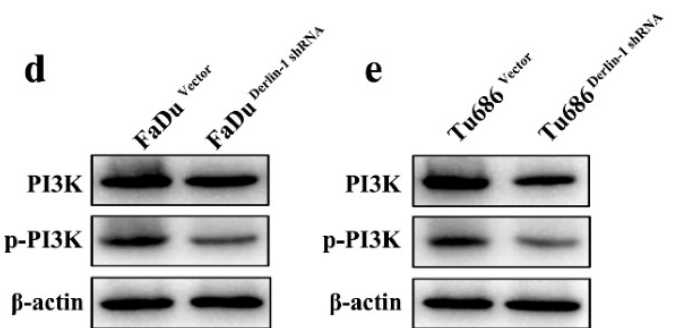

b
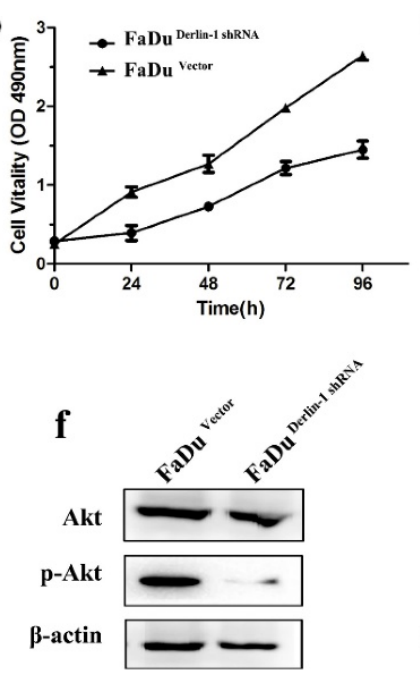
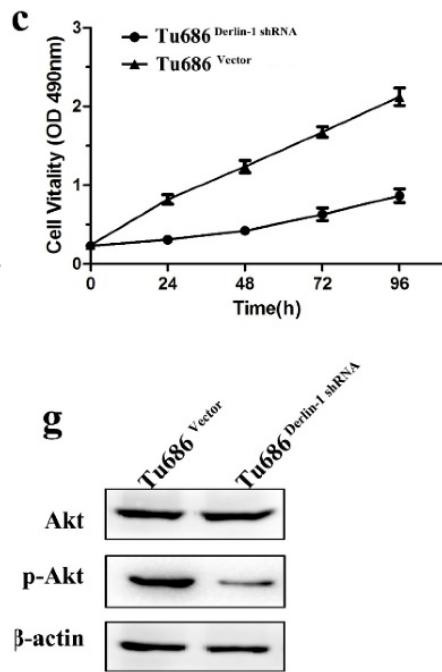

Figure 3: Decreased the expression of Derlin-1 inhibited SCCHN cell proliferation and PI3K/Akt activation. FaDu and Tu686 cell lines were transiently transfected with shRNA control or shRNA Derlin-1. (a). Derlin-1 shRNA was successes to knock down the expression of Derlin-1 in FaDu and Tu686 cell lines. (b, c). A CCK-8 assay was performed to show that knock down Derlin-1 expression could inhibit the FaDu and Tu686 cell viability on 4 time points. (d-g). Following transient transfected with shRNA control or shRNA Derlin-1 for 48h, the total and phosphorylated of PI3K and Akt expression were significantly reduced in FaDu and Tu686 cells with Derlin-1 down-regulation.

a
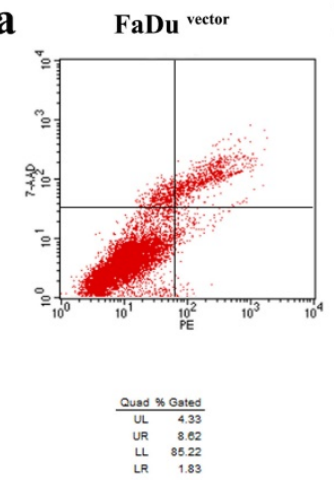

b FaDu Derlin-1 shRNA

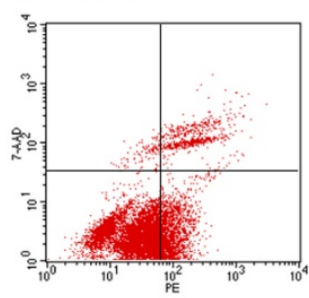

\begin{tabular}{cc} 
Ougd \% Gsted \\
\hline$U L$ & 1.27 \\
UR & 8.52 \\
LL & 71.30 \\
LR & 20.91
\end{tabular} c

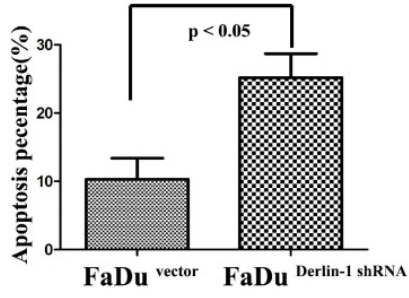

g

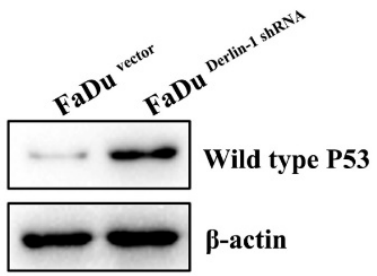

d

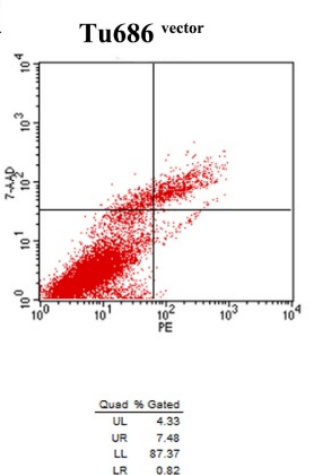

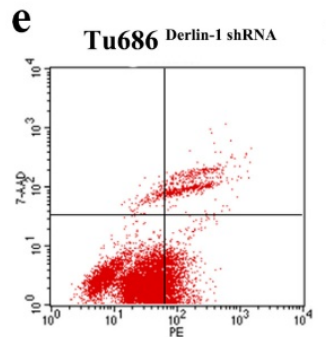
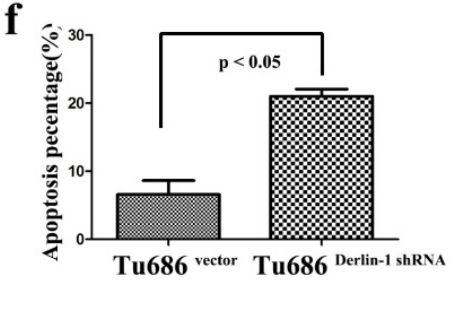

h

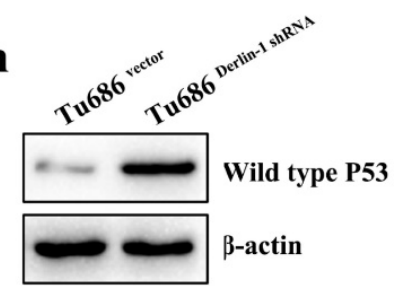

Figure 4: Down regulation of Derlin-1 induced SCCHN cell apoptosis and p53 expression. (a-b; d-e). Flow cytometry analysis showed that apoptotic percentages of FaDu and Tu686 cells were increased in the group of Derlin-1 with transient transfection for $48 \mathrm{~h}$. (c, f). The quantitative analysis for apoptotic FaDu and Tu686 cells respectively showed that the difference was significant $(\mathrm{p}<0.05)$. $(\mathbf{g}, \mathbf{h})$. The expression of wild type p53 was promoted in both of FaDu and Tu686 cells when Derlin-1 shRNA was transfected transiently for $48 \mathrm{~h}$.

\section{Decreased expression of Derlin-1 increased apoptosis of SCCHN cell lines}

Apoptosis was measured in both Tu686 and FaDu cell line with Derlin-1 knock down. As present- ed in Figure 4a-f, the percentages of early and late apoptotic cells were significantly increased in Tu686 and FaDu cell line with Derlin-1 knock down $(p<0.05)$. Interestingly, p53 was induced by Derlin-1 downregulation in Tu686 and FaDu cell line (Figure 4g-h). 

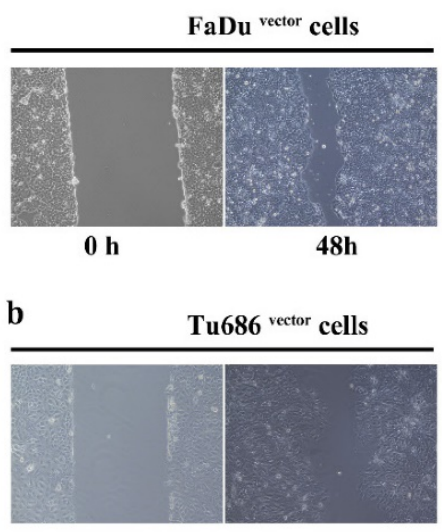

O h

e

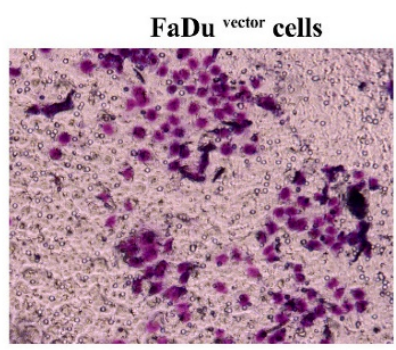

f

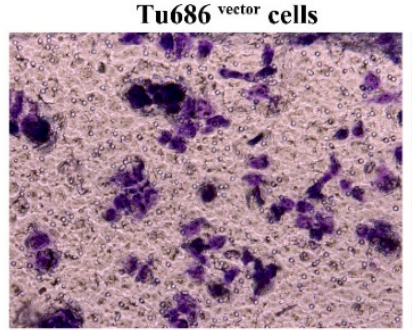

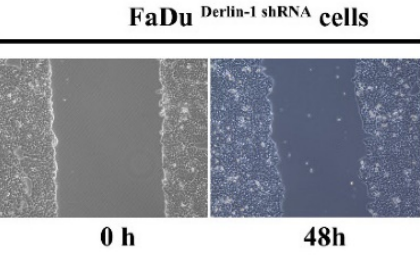

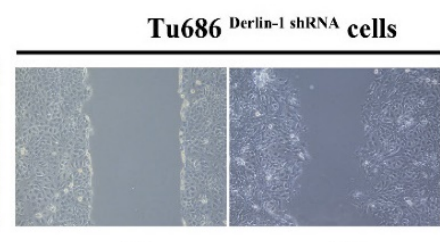

$\mathbf{0 ~ h}$

$48 \mathrm{~h}$

FaDu Derlin-1 shRNA cells

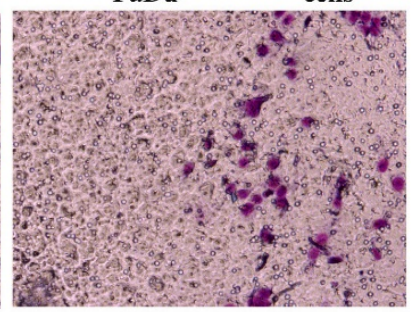

Tu686 Derlin-1 shRNA cells

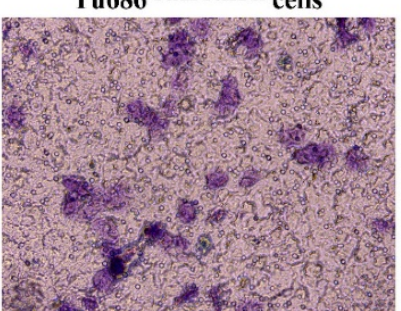

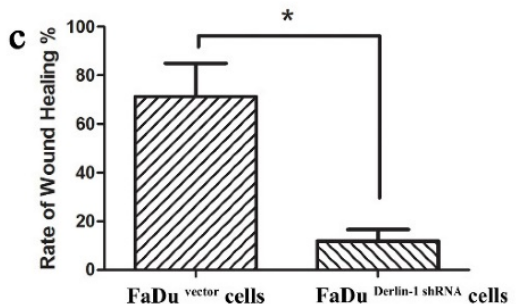

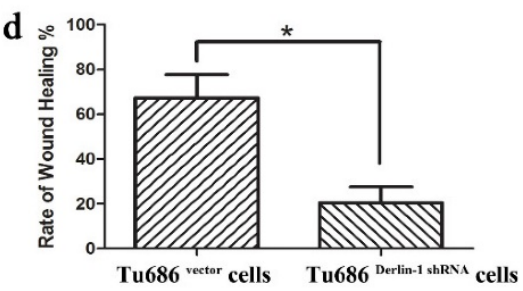

f

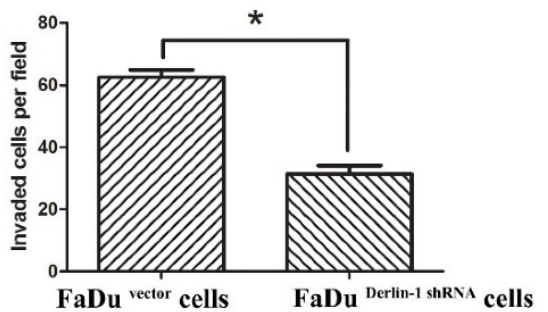

g

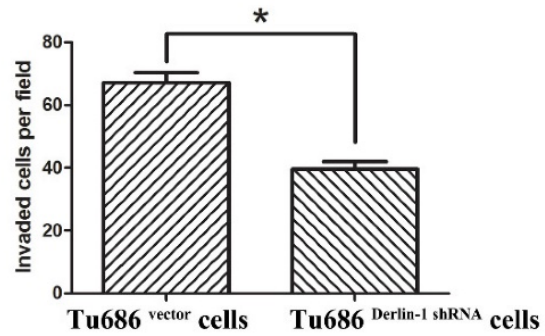

Figure 5: Inhibition of Derlin-1 attenuated the capability of SCCHN cell wound healing and migration. (a, b). Wound healing experiment showed that there was a different healing ratios between the group of vector and Derlin-1 shRNA in FaDu cell as well as Tu686 cell following by transient transfected for $48 \mathrm{~h}$. (c, d). The quantitative analysis for the ratio of wound healing in FaDu and Tu686 cell lines respectively showed that the difference was significant (asterisk means $p<0.05)$. (e, f). Transwell experiment showed that the invaded cell number in the group of FaDu and Tu686 cells with transient transfection of vector and Derlin-1 shRNA for $48 \mathrm{~h}$. (g, h). The quantitative analysis for $\mathrm{FaDu}$ and Tu686 cell invasion respectively showed that the difference was significant (asterisk means $\mathrm{p}<0.05$ ).

\section{Knock down expression of Derlin-1 weaken the metastatic capability of SCCHN cell lines}

Metastasis was thought of a lethal factor in various cancer patients. Considering our above result indicated that Derlin-1 expression was associated with lymph node metastasis, the invasion and migration of SCCHN cell lines were compared in the groups of Derlin-1 knock down and corresponding control. Wound healing experiment showed that cells transfected Derlin-1 shRNA migrated slower than control cells $(71.37 \pm 13.55 \%$ vs $11.81 \pm 4.72 \%$; $67.32 \pm$ $10.31 \%$ vs $20.46 \pm 7.18 \%$, p $<0.05$, Figure $5 a-b)$. The similar tendency was also observed in the transwell experiment (Figure 5e-f), which means dysregulation of Derlin-1 mediated cell invasion. Our previous work indicated that TGF- $\beta 1$ promoted SCCHN cell metastasis via epithelial-mesenchymal transition ${ }^{14}$. As showed in Figure 6a, the epithelial biomarker E-cadherin was up-regulated in Derlin-1 knock down cells, which were accompanied by the downregulation of Vimentin (mesenchymal biomarker). Furthermore, both of Smad2/3 and phosphorylated Smad2/3 were decreased in Tu686 and FaDu cell line with Derlin-1 knock down (Figure 6b).

\section{Discussion}

This study found that Derlin-1 protein was over-expressed in SCCHN cell lines and cancer tissues comparing to non-transformed immortalised epithetlium cell line and adjacent normal mucosa respect- 
ively. It kept according with investigations in lung11, breast $^{8}$ and colon $^{9}$ cancers. Endoplasmic reticulum (ER) was a crucial platform coordinating several signaling including proliferation, apoptosis and migration ${ }^{15,16}$. ER stress usually was evoked and accumulated when uncontrolled growth of SCCHN cells encountering unfavorable conditions such as hypoxia, chemo-/radio-therapy and $\mathrm{pH}$ alteration in microenvironment ${ }^{17}$. It was reported that Derlin-1 was up-regulated in breast cancer cells and yeast when ER-stress was activated 8,18 . Therefore, the over-expression of Derlin-1 in SCCHN may be caused by ER-stress activation in this solid tumor. Furtherly, we found that Derlin-1 expression was associated with lymph node metastasis, recurrence and clinical stage, which meant Derlin-1 played a vital role in the initiation and progression of SCCHN. Importantly, the research implied that Derlin-1 expression could be an independently prognostic factor and its high expression significantly involved to a shorter survival time of SCCHN patients. Taking together with similar discoveries of other labs in breast ${ }^{8}$, colon ${ }^{9}$ and lung 11 cancers, Derlin- 1 could be thought of an important biomarker of cancer prognosis and worth to further investigation.

The primary function of ER was help to fold and mature the secretory and transmembrane proteins ${ }^{19}$. Protein would be retro-translocated to cytosol from ER when adversely intrinsic and extrinsic alternation make it misfolded or immaturity ${ }^{20}$. Then, the unfolded protein response (UPR), or named ER associated degradation (ERAD), would preferentially degrade the improper protein ${ }^{7}$. In the early stage of ER-stress activation, cell growth was promoted ${ }^{21}$. However, prolonged ER-stress would induce apoptosis if these disadvantageous stimulations persist, especially in the late stage of ER-activation ${ }^{21}$. The balance of pro-survival and pro-apoptosis controlled by UPR determined cell fate. Derlin-1 was firstly found in 2004 and confirmed as one component of complex for misfolded protein retro-translocation ${ }^{4}$. Whether the UPR associated protein, Derlin-1, regulated proliferation of SCCHN cells was investigated in this study. Our experiments indicated that knock down Derlin-1 expression could inhibit growth of both Tu686 and FaDu cell lines. And, we found PI3K/Akt pathway was involved in the Derlin-1 knock down cells. It is noteworthy that PI3K/Akt signaling plays a distinctly pro-survival role in multifaceted ways $^{22}$. When Derlin-1 protein was down-regulated in Tu686 and FaDu cell lines, phosphorylation of PI3K was reduced significantly, which was also reported in colon cancer cell line. Although the detailed mechanism of Derlin-1 regulating PI3K/Akt remains unclear, the possible reason may be that related to ERAD.
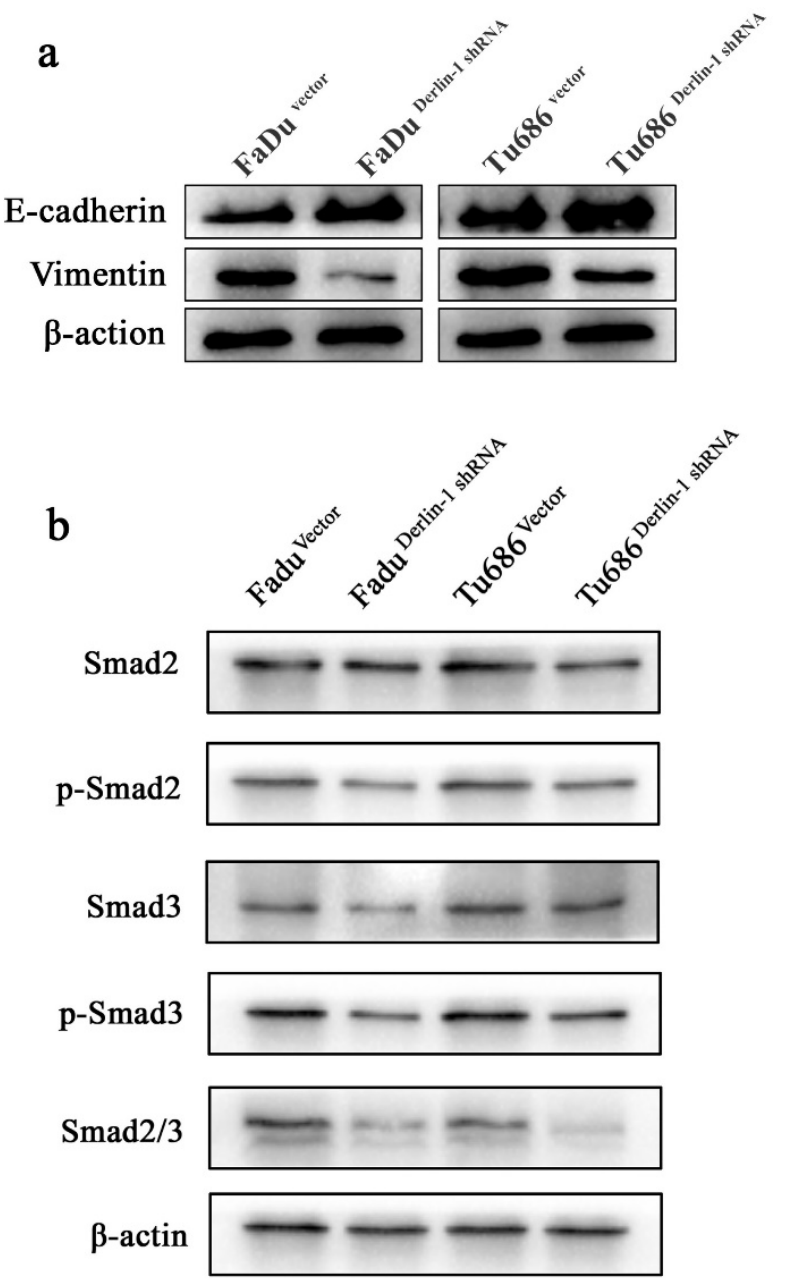

Figure 6: Derlin-1 regulated EMT related protein expression in SCCHN. (a). The expression of E-cadherin was induced, while Vimentin expression was depressed, in FaDu and Tu686 cells with Derlin-1 knock down transiently comparing to the vector control for $48 \mathrm{~h}$. (b). The total and phosphorylated of Smad2 and Smad3 expression were significantly reduced in $\mathrm{FaDu}$ and Tu686 cells with Derlin-1 down-regulation transiently for $48 \mathrm{~h}$.

Apoptosis escape was another cause of cancer permanent growth ${ }^{23}$. Derlin-1 could constitute a part of retro-translocation channel for ERAD 5 . It was well known that ERAD generally inhibited apoptosis if ER stress was not severe and prolonged ${ }^{21}$. Down-regulation of Derlin-1 in cancer cells may weaken the ability of ERAD and then promote cell apoptosis. This study exactly illustrated that knock down Derlin-1 expression could increase the cell apoptosis of Tu686 and FaDu cell line. Meanwhile, our result showed that p53 protein was promoted in Tu686 and FaDu cells with Derlin-1 knockdown. The p53 tumor suppressor gene plays the vital role in maintaining the genetic homogeneity of somatic cells and is often inactivated in cancer cells ${ }^{24}$. ER stress targeted wild-type p53 into the cytoplasm, where it is 
degraded 25 , providing a hypothesis for the inhibition of the apoptotic activity by ERAD-p53 pathway. Certainly, whether there is another mechanism of Derlin-1 regulating p53 protein expression required further study.

Interestingly, we found that decreased expression of Derlin-1could reduce the migration and invasion of SCCHN cell lines, which happened to coincide with our above result that Derlin-1 expression associated with lymph node metastasis of SCCHN. And, the similar result in canine mammary adenocarcinomas ${ }^{26}$ and human lung cancer ${ }^{11}$ double confirmed the possibility of Derlin-1 regulating cancer cell metastasis. Our previous work indicated that TGF- $\beta 1$ moderated SCCHN metastasis by induction of epithelial-mesenchymal transition(EMT) ${ }^{14}$. In the process of EMT, loss biomarker of the cell-cell adhesion, such as E-cadherin, is controlled by some transcription regulators such as Snail-1, Slug and Twist that can promote mesenchymal characteristics including expression of vimentin, fibronectin, and $\mathrm{N}$-cadherin ${ }^{27}$. Here, we found that down-regulation of Derlin-1 expression could reduce the mesenchymal biomarker (Vimentin) expression and promote the epithelial biomarker (E-cadherin) expression. Meanwhile, the expression of Smad2/3 and their phosphorylated proteins were significantly decreased when Derlin-1 expression was knock down in Tu686 and $\mathrm{FaDu}$ cell lines. Phosphorylated Smad2/3 could facilitate the translocation of Smad4 into the nucleus where the complex can regulate transcription of its target genes such as E-cadherin ${ }^{28}$. We noticed that the invasion assay in our study did not normalized by cell number reduction. Therefore, we can't exclude the possibility of that Derlin-1 induced cell invasion by mediating apoptosis. And, the possible mechanism of Derlin-1 regulating SCCHN cell metastasis may be related to both of EMT and apoptosis escape.

In conclusion, this study indicated that Derlin-1 was significantly over-expressed in SCCHN samples and associated with prognosis of SCCHN patients. What more, knock down expression of Derlin-1 in SCCHN cell lines could promote apoptosis and inhibit cell proliferation and metastasis. In spite of further function gain and loss studies required, PI3K/Akt, p53 and Smad2/3 signaling may involve in these processes regulated by Derlin-1. Taking together, Derlin-1 appears to be a promising biomarker which could be targeted for therapy, particularly in SCCHN patients with Derlin-1 overexpression.

\section{Acknowledgements}

This study was funded by grants from the National Natural Science Foundation of China (No. $81602389,81472696,81202128,81272974)$ and the
Natural Science Foundation of Hunan Province (No. 2017JJ3456, 2015JJ3137).

\section{Contributions}

LM Pi, YQ Tian and $X$ Zhang designed this study. LM Pi performed all experiments. L She, M Wei, GC Liu, CH Chen, HL Tan assisted with experimental performance. DH Huang, YQ Tian assisted with the data analysis. LM Pi, GC Zhu, Y Liu and $X$ Zhang wrote and modified the manuscript.

\section{Competing Interests}

The authors declare no conflict of interest.

\section{References}

1. Torre LA, Bray F, Siegel RL, Ferlay J, Lortet-Tieulent J, Jemal A. Global cancer statistics, 2012. CA: a cancer journal for clinicians. 2015;65(2):87-108.

2. Siegel RL, Miller KD, Jemal A. Cancer statistics, 2016. CA: a cancer journal for clinicians. 2016;66(1):7-30.

3. Russo N, Wang X, Liu M, et al. A novel approach to biomarker discovery in head and neck cancer using an autoantibody signature. Oncogene. 2013;32(42):5026-5037.

4. Lilley BN, Ploegh HL. A membrane protein required for dislocation of misfolded proteins from the ER. Nature. 2004;429(6994):834-840.

5. Ye Y, Shibata Y, Yun C, Ron D, Rapoport TA. A membrane protein complex mediates retro-translocation from the ER lumen into the cytosol. Nature. 2004;429(6994):841-847.

6. Hampton RY. ER stress response: getting the UPR hand on misfolded proteins. Current biology: CB. 2000;10(14):R518-521.

7. Wang M, Kaufman RJ. The impact of the endoplasmic reticulum protein-folding environment on cancer development. Nature reviews Cancer. 2014;14(9):581-597.

8. Wang J, Hua H, Ran Y, et al. Derlin-1 is overexpressed in human breast carcinoma and protects cancer cells from endoplasmic reticulum stress-induced apoptosis. Breast cancer research: BCR. 2008;10(1):R7.

9. Tan X, He X, Jiang Z, et al. Derlin- 1 is overexpressed in human colon cancer and promotes cancer cell proliferation. Molecular and cellular biochemistry. 2015;408(1-2):205-213.

10. Wu Z, Wang C, Zhang Z, et al. High Expression of Derlin-1 Is Associated with the Malignancy of Bladder Cancer in a Chinese Han Population. PloS one. 2016;11(12):e0168351.

11. Dong QZ, Wang Y, Tang ZP, et al. Derlin-1 is overexpressed in non-small cell lung cancer and promotes cancer cell invasion via EGFR-ERK-mediated up-regulation of MMP-2 and MMP-9. The American journal of pathology. 2013;182(3):954-964.

12. Zhu GC, Liu LJ, She L, et al. Elevated expression of histone demethylase PHF8 associates with adverse prognosis in patients of laryngeal and hypopharyngeal squamous cell carcinoma. Epigenomics-Uk. 2015;7(2):143-153.

13. Zhu GC, Yu CY, She L, et al. Metadherin Regulation of Vascular Endothelial Growth Factor Expression Is Dependent Upon the PI3K/Akt Pathway in Squamous Cell Carcinoma of the Head and Neck. Medicine. 2015;94(6).

14. Yu C, Liu Y, Huang D, et al. TGF-beta1 mediates epithelial to mesenchymal transition via the TGF-beta/Smad pathway in squamous cell carcinoma of the head and neck. Oncology reports. 2011;25(6):1581-1587.

15. Ma D, Zerangue N, Lin YF, et al. Role of ER export signals in controlling surface potassium channel numbers. Science. 2001;291(5502):316-319.

16. Hotamisligil GS, Davis RJ. Cell Signaling and Stress Responses. Cold Spring Harbor perspectives in biology. 2016;8(10).

17. Chiu CC, Lin CY, Lee LY, et al. Molecular chaperones as a common set of proteins that regulate the invasion phenotype of head and neck cancer. Clinical cancer research: an official journal of the American Association for Cancer Research. 2011;17(14):4629-4641.

18. Travers KJ, Patil CK, Wodicka L, Lockhart DJ, Weissman JS, Walter P. Functional and genomic analyses reveal an essential coordination between the unfolded protein response and ER-associated degradation. Cell. 2000;101(3):249-258

19. Lee AS, Hendershot LM. ER stress and cancer. Cancer biology \& therapy. 2006;5(7):721-722.

20. McCaffrey $\mathrm{K}$, Braakman I. Protein quality control at the endoplasmic reticulum. Essays in biochemistry. 2016;60(2):227-235.

21. Rao RV, Ellerby HM, Bredesen DE. Coupling endoplasmic reticulum stress to the cell death program. Cell death and differentiation. 2004;11(4):372-380.

22. Naguib A. Following the trail of lipids: Signals initiated by PI3K function at multiple cellular membranes. Science signaling. 2016;9(428):re4.

23. Papa S, Bubici C. Linking apoptosis to cancer metabolism: Another missing piece of JuNK. Molecular \& cellular oncology. 2016;3(2):e1103398. 
24. Seton-Rogers S. Genetics: p53 variant increases cancer risk. Nature reviews Cancer. 2016;16(5):273.

25. Stavridi ES, Halazonetis TD. p53 and stress in the ER. Genes $\mathcal{E}$ development. 2004;18(3):241-244.

26. Klopfleisch R, Klose P, Gruber AD. The combined expression pattern of BMP2, LTBP4, and DERL1 discriminates malignant from benign canine mammary tumors. Veterinary pathology. 2010;47(3):446-454.

27. Seton-Rogers S. Epithelial-mesenchymal transition: Untangling EMT's functions. Nature reviews Cancer. 2016;16(1):1.

28. Hill CS. Transcriptional Control by the SMADs. Cold Spring Harbor perspectives in biology. 2016;8(10). 